\title{
$\beta$ Cell dysfunction exists more than 5 years before type 1 diabetes diagnosis
}

\author{
Carmella Evans-Molina, ${ }^{1,2,3,4,5,6}$ Emily K. Sims, ${ }^{4,5}$ Linda A. DiMeglio, ${ }^{4,5}$ Heba M. Ismail, ${ }^{7}$ \\ Andrea K. Steck, ${ }^{8}$ Jerry P. Palmer, ${ }^{9}$ Jeffrey P. Krischer, ${ }^{10}$ Susan Geyer, ${ }^{10}$ Ping Xu, ${ }^{10}$ Jay M. Sosenko, ${ }^{11}$ \\ and the Type 1 Diabetes TrialNet Study Group ${ }^{12}$ \\ 1Departments of Medicine, ${ }^{2}$ Cellular and Integrative Physiology, ${ }^{3}$ Biochemistry and Molecular Biology, ${ }^{4}$ Pediatrics, and \\ the ${ }^{5}$ Herman B Wells Center for Pediatric Research, Indiana University School of Medicine, Indianapolis, Indiana, USA \\ ${ }^{6}$ Roudebush VA Medical Center, Indianapolis, Indiana, USA. 'Children's Hospital of Pittsburgh of UPMC, University of \\ Pittsburgh, Pittsburgh, Pennsylvania, USA. ${ }^{8}$ Barbara Davis Center for Childhood Diabetes, University of Colorado School \\ of Medicine, Denver, Colorado, USA. ${ }^{9}$ VA Puget Sound Health Care System and the University of Washington, Seattle, \\ Washington, USA. ${ }^{10} \mathrm{Health}$ Informatics Institute, University of South Florida, Tampa, Florida, USA. "Department of \\ Medicine and the Diabetes Research Institute, Leonard Miller School of Medicine, University of Miami, Miami, Florida, \\ USA. ${ }^{12}$ The Type 1 Diabetes TrialNet Study Group is detailed in the supplemental acknowledgments.
}

BACKGROUND. The duration and patterns of $\beta$ cell dysfunction during type 1 diabetes (T1D) development have not been fully defined.

METHODS. Metabolic measures derived from oral glucose tolerance tests (OCTTs) were compared between autoantibody-positive $(\mathrm{aAb}+)$ individuals followed in the TrialNet Pathway to Prevention study who developed diabetes after 5 or more years or less than 5 years of longitudinal follow-up (Progressors $\geq 5, n=75$; Progressors $<5, n=474$ ) and $144 \mathrm{aAb}$-negative (aAb-) relatives.

RESULTS. Mean age at study entry was $15.0 \pm 12.6$ years for Progressors $\geq 5 ; 12.0 \pm 9.1$ for Progressors $<5$; and $16.3 \pm 10.4$ for aAb- relatives. At baseline, Progressors $\geq 5$ already exhibited significantly lower fasting C-peptide $(P<0.01)$, C-peptide AUC $(P<0.001)$, and early C-peptide responses (30- to 0 -minute $C$-peptide; $P<0.001$ ) compared with aAb-relatives, while 2 -hour glucose $(P=0.03)$, glucose AUC $(<0.001)$, and Index60 $(<0.001)$ were all higher. Despite significant baseline impairment, metabolic measures in Progressors $\geq 5$ were relatively stable until 2 years prior to T1D diagnosis, when there was accelerated C-peptide decline and rising glycemia from 2 years until diabetes diagnosis. Remarkably, patterns of progression within 3 years of diagnosis were nearly identical between Progressors $\geq 5$ and Progressors $<5$.

CONCLUSION. These data provide insight into the chronicity of $\beta$ cell dysfunction in T1D and indicate that $\beta$ cell dysfunction may precede diabetes diagnosis by more than 5 years in a subset of $a A b+$ individuals. Even among individuals with varying lengths of aAb positivity, our findings indicate that patterns of metabolic decline are uniform within the last 3 years of progression to T1D.

TRIAL REGISTRATION. Clinicaltrials.gov NCT00097292.

FUNDING. The Type 1 Diabetes TrialNet Study Group is a clinical trials network currently funded by the NIH through the National Institute of Diabetes and Digestive and Kidney Diseases, the National Institute of Allergy and Infectious Diseases, and The Eunice Kennedy Shriver National Institute of

Conflict of interest: The authors have declared that no conflict of interest exists.

Submitted: March 14, 2018 Accepted: June 21, 2018 Published: August 9, 2018

\section{Reference information:} JCI Insight. 2018;3(15):e120877. https://doi.org/10.1172/jci. insight.120877.
Child Health and Human Development and the Juvenile Diabetes Research Foundation.

\section{Introduction}

The pathogenesis of type 1 diabetes (T1D) involves the development of $\beta$ cell autoimmunity, marked by the development of circulating autoantibodies (aAbs) against $\beta$ cell antigens, and declining insulin secretion. Longitudinal findings from the Diabetes Prevention Trial - Type 1 (DPT-1) showed that aAb-positive $(\mathrm{Ab}+)$ individuals had an acceleration in the decline of the first-phase insulin response (1), the early C-peptide response (30- to 0 -minute difference) (2), and $\beta$ cell glucose sensitivity (3) $1-2$ years prior to the diagnosis of T1D. However, since the DPT-1 study mostly followed individuals within 3 years of diagnosis, little is 
known about patterns of metabolic decline in $\mathrm{aAb}+$ individuals over longer periods of follow-up. Analysis of data from individuals who underwent extended observation prior to diagnosis would yield information about the potential chronicity of metabolic abnormalities and lend further insight into long-term patterns of metabolic decline during early stages of T1D.

Since 2004, over 180,000 relatives of individuals with T1D have been screened for the presence of islet aAbs as part of the international Type 1 Diabetes TrialNet Pathway to Prevention (PTP) study. Over 5,000 individuals have been identified as $\mathrm{aAb}+$ and enrolled for longitudinal monitoring with either annual or biannual oral glucose tolerance testing, providing a unique opportunity to analyze natural history data from a large and geographically diverse at-risk $\mathrm{aAb}+$ cohort of individuals with variable and often long durations of follow-up (4). Within this cohort, we studied a subset of $75 \mathrm{aAb}+$ individuals who progressed to T1D after a minimum of 5 years of follow-up. This length of follow-up was conducive for assessing the chronicity of $\beta$ cell dysfunction prior to diagnosis and provided an opportunity to study patterns of $\beta$ cell deterioration over a much longer period of progression compared with prior natural history studies.

\section{Results}

Progressors with 5 or more years of follow-up (Progressors $\geq 5$ ) were monitored for $6.6 \pm 1.3$ years from baseline oral glucose tolerance test (OGTT) to T1D diagnosis. They represented $13.7 \%$ (75 of 549) of all analyzed T1D cases within the study cohort: $12 \%$ were single aAb+, and $88 \%$ were multiple aAb+at enrollment (Figure 1 and Supplemental Table 1; supplemental material available online with this article; https://doi.org/10.1172/jci.insight.120877DS1).

Comparison of baseline metabolic measures between Progressors $\geq 5$ and $a A b-$ relatives. To assess the duration and extent of $\beta$ cell dysfunction prior to the onset of T1D, we compared baseline metabolic data between $75 \mathrm{aAb}+$ Progressors $\geq 5$ and $144 \mathrm{aAb}$-negative relatives $(\mathrm{aAb}-)$ who agreed to undergo OGTT testing after negative screening for aAbs. Supplemental Table 1 shows the baseline characteristics of the Progressors $\geq 5$ and $\mathrm{aAb}$ - participants. Progressors $\geq 5$ were younger $(P=0.01$ for pairwise comparison) and had lower BMI $Z$-score values ( $P=0.03$ for pairwise comparison). Otherwise, there were no significant differences in gender, race, ethnicity, or relationship to the T1D proband. At their baseline OGTTs, after adjustments for age and BMI $Z$ score, Progressors $\geq 5$ had already demonstrated significant reductions in the fasting $C$-peptide $(P<0.01)$, the early C-peptide response $(P<0.001)$, and C-peptide AUC $(P<0.001)$ compared with the $\mathrm{aAb}-$ group. Consistent with these changes, the glucose AUC $(P<0.001)$, 2-hour glucose $(P=0.03)$, and Index60 $(P<0.001)$ were all significantly higher in Progressors $\geq 5$. Fasting glucose levels did not differ between the groups (Table 1$)$.

Longitudinal patterns of metabolic decline in Progressors $\geq 5$. Figure 2 shows the mean values of the fasting C-peptide, early C-peptide response, C-peptide AUC, fasting glucose, 2-hour glucose, glucose AUC, and Index60 at baseline and then each year ( \pm 6 months) from 4 years to diagnosis for Progressors $\geq 5$. Also shown for reference are mean values of the $\mathrm{aAb}$ - individuals at baseline (red dotted line). Again, evident are the marked differences at baseline between Progressors $\geq 5.0$ and $\mathrm{AAb}$-participants. Although the numbers vary between the time points, the figure suggests a consistent pattern of metabolic decline. This pattern was notable for the presence of substantial metabolic abnormalities that were already present on average $6.6 \pm 1.3$ years before diagnosis. However, there was relatively little change in these metabolic indices until approximately 2 years before diagnosis. Marked decreases in C-peptide and increases in glucose then became apparent, especially during the last year before diagnosis. An exception was the C-peptide AUC, which tended to increase after baseline and then declined just prior to diagnosis.

To examine this pattern quantitatively, we analyzed the absolute and percentage changes from baseline to 4 years before diagnosis, 4 years to 1 year before diagnosis, and 1 year to diagnosis (Table 2 ). The analysis included only those with paired OGTTs at the boundaries for each interval. From baseline (6.6 \pm 1.3 years) to 4 years, the fasting glucose $(P=0.02)$ and glucose AUC $(P=0.03)$ increased significantly. However, there were no significant changes in the early C-peptide response, the C-peptide AUC, or the Index60 during this time period. Counter to what might be expected, the fasting C-peptide increased $(P=0.001)$.

More appreciable changes in the selected metabolic indices were observed between 4 years and 1 year prior to diagnosis (Table 2). During this time period, all measures of glycemia significantly increased, including the fasting glucose $(P=0.01)$, 2-hour glucose $(P=0.002)$, glucose AUC $(P=0.01)$, and Index60 $(P=0.004)$. The fasting $C$-peptide continued to increase $(P=0.01)$; however, there was no significant change in the early $\mathrm{C}$-peptide response or the C-peptide AUC. 


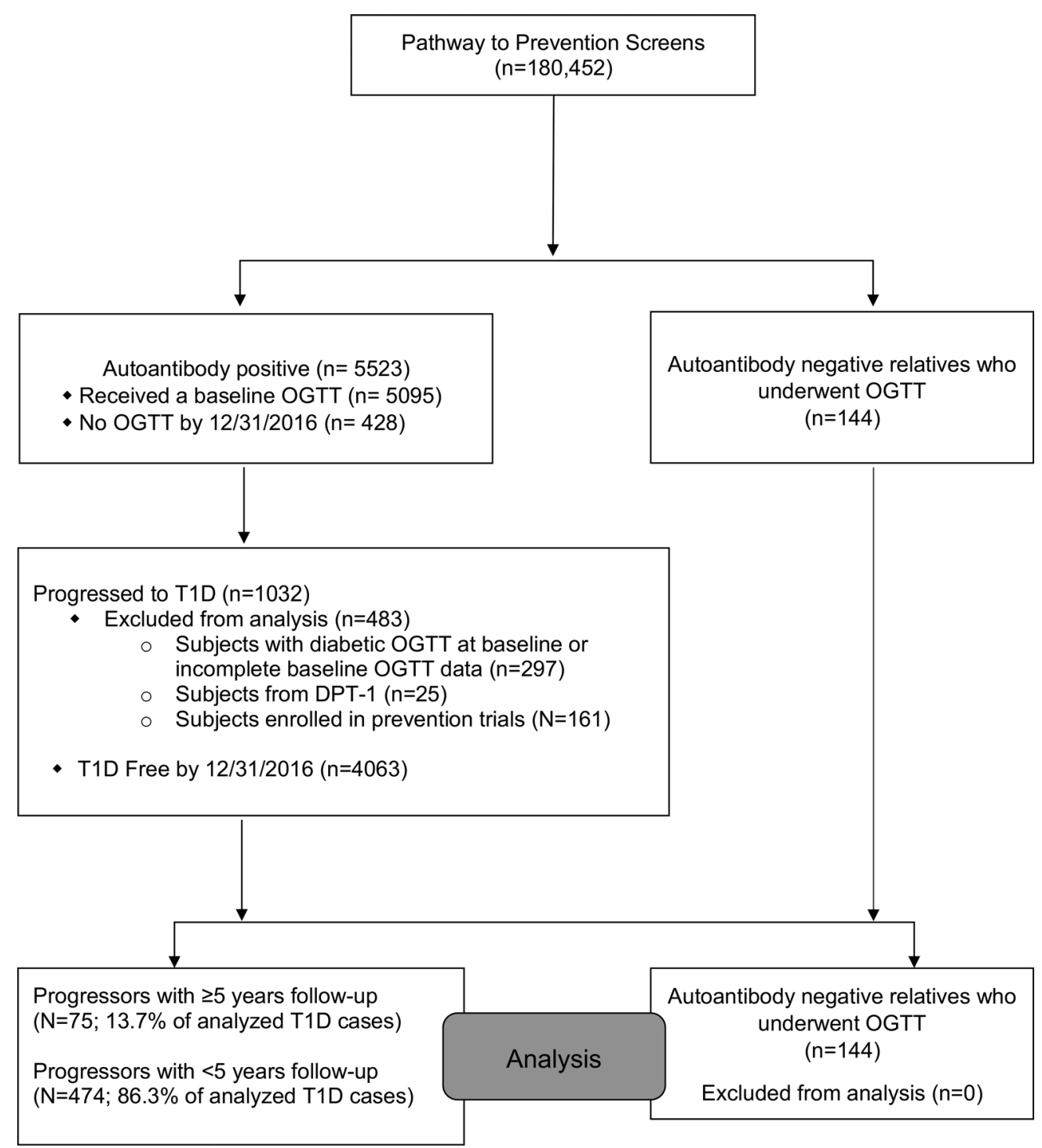

Figure 1. Participant flow through the Type 1 Diabetes TrialNet Pathway to Prevention Study.

As shown in Figure 2, there was marked metabolic deterioration from 1 year prior to the time of diagnosis (Table 2). During this terminal period, the early $\mathrm{C}$-peptide response $(P<0.001)$ and the C-peptide AUC $(P=0.01)$ significantly declined, while the fasting glucose, 2-hour glucose, glucose AUC, and Index60 (all $P<0.001$ ) significantly increased. The fasting C-peptide continued to increase, but not to a significant extent $(P=0.13)$.

Comparisons of changes between Progressors $\geq 5$ and Progressors followed less than 5 years. To address whether patterns of $\beta$ cell dysfunction were fundamentally different between Progressors $\geq 5$ and aAb+ PTP participants with less than 5 years of follow-up (Progressors $<5$ ), we compared metabolic indices between these groups from study entry until T1D diagnosis. Compared with Progressors $<5$, Progressors $\geq 5$ were older at study entry $(15.0 \pm 12.6$ years vs. $12.0 \pm 9.1$ years; $P=0.01)$ and at diagnosis $(21.6 \pm 12.8$ years vs. $13.9 \pm$ 9.3 years; $P<0.001$ ). The mean follow-up for Progressors $\geq 5$ was $6.6 \pm 1.3$ years, compared with a mean follow-up for Progressors $<5$ of $1.9 \pm 1.3$ years. Progressors $\geq 5$ had a lower BMI $Z$ score at study entry with a median value of 0.41 (interquartile range [IQR]: -0.31 to 1.14 ) versus Progressors $<5$ who had a median BMI $Z$ score of 0.69 (IQR: 0.04 to $1.46 ; P=0.04$ for pairwise comparison). The percentage of Progressors $\geq 5$ and Progressors $<5$ who were positive for a single $\mathrm{aAb}$ or were multiple-aAb positive at study entry was similar. Supplemental Table 2 shows comparisons of metabolic data at study entry for Progressors $\geq 5$ and Progressors $<5$. As expected since Progressors $<5$ were closer to T1D diagnosis, they exhibited significantly 
Table 1. Comparison of metabolic parameters between Progressors $\geq 5$ and aAb- relatives at study entry

\begin{tabular}{|c|c|c|c|c|}
\hline & Progressors $\geq 5(n=75)$ Mean (SD) & aAb- Controls $(n=144)$ Mean (SD) & $P$ value $^{A}$ & $P$ value ${ }^{B}$ \\
\hline Early C-peptide Response (ng/ml) & $2.95(1.65)$ & $4.89(2.29)$ & $<0.001$ & $<0.001$ \\
\hline Fasting Glucose (mg/dl) & $87.31(9.07)$ & $89.37(7.38)$ & 0.09 & 0.11 \\
\hline 2-hour Glucose (mg/dl) & $118.84(28.64)$ & $111.14(25.9)$ & 0.045 & 0.03 \\
\hline Glucose AUC & $136.2(22.02)$ & $125.6(23.94)$ & 0.01 & $<0.001$ \\
\hline
\end{tabular}

${ }^{A}$ Student's $t$ test. ${ }^{B}$ Multivariate linear regression with age and BMI $Z$ scores as covariates. Index 60 values were log transformed for comparisons. AUC, area under the curve; aAb-, autoantibody-negative controls; SD, standard deviation.

lower fasting C-peptide levels $(P=0.048)$ and higher fasting glucose $(P=0.005)$, 2-hour glucose $(P<0.001)$, glucose AUC $(P=0.002)$, and Index60 values $(P=0.002)$. However, no differences were observed in the C-peptide AUC or early C-peptide responses.

To test whether longitudinal patterns of metabolic decline were different between these 2 groups, mean values of metabolic measures at 3 years ( \pm 6 months), 2 years ( \pm 6 months), and 1 year ( \pm 6 months) prior to T1D diagnosis were compared in the Progressors $\geq 5$ and Progressors $<5$ (Table 3). Remarkably, there were no significant differences in the mean values of fasting glucose, 2-hour glucose, glucose AUC, fasting C-peptide, C-peptide AUC, early C-peptide response, or Index60 between the 2 groups at any of the time points tested. Next, we compared the absolute and percentage changes in these metabolic measures between Progressors $\geq 5$ and Progressors $<5$ from 3 years to 1 year before diagnosis and from 1 year to diagnosis. Similar to analysis of mean values (Table 3), no significant difference in either the absolute or percentage change for each of the analyzed metabolic measures was observed between the 2 groups (Supplemental Table 3).

Finally, to address whether severity of metabolic dysfunction was different between these groups at the time of T1D diagnosis, mean values of the same metabolic variables were compared in Progressors $\geq 5$ and Progressors $<5$ who underwent an OGTT at the time of T1D diagnosis. Fasting C-peptide and C-peptide AUC values were lower in the Progressors $<5(P<0.001)$. However, following adjustment for age and BMI $Z$ score, no significant differences in any of the measures were observed. (Supplemental Table 4).

\section{Discussion}

Findings from this analysis of aAb+ individuals followed longitudinally for 5 or more years in the TrialNet PTP study can be viewed according to 3 metabolic phases of T1D progression. The first phase was marked by appreciable metabolic impairment at baseline (on average over 6 years before diagnosis), especially with regard to early C-peptide indices. It is unclear, however, when these abnormalities first occur, as subjects were recruited into the cohort after the development of aAb positivity. During the second phase, spanning from study entry to approximately 2 years before diagnosis, there was relatively little change in either C-peptide or glucose indices. Finally, a third phase was evident during the last 2 years, in which there was accelerated metabolic deterioration, characterized by rising glycemia and worsening C-peptide secretion. Since the 3-phase model could be a function of the study population, data limitations, and the analytic construct, it is not definitive. Still, a consideration of the model raises several fundamental questions regarding the pathogenesis of T1D.

First metabolic phase. At study entry, Progressors $\geq 5$ already demonstrated significant differences in the majority of C-peptide and glucose measures. Especially prominent were impairments in the early C-peptide response, which were approximately $40 \%$ lower in Progressors $\geq 5$ compared with the $\mathrm{aAb}$ - controls. Similar findings were seen in the Type I Diabetes Prediction and Prevention (DIPP) study, which followed Finnish children with high-risk HLA alleles before and after seroconversion. Intravenous GTTs performed at the time of initial seroconversion showed that $42 \%$ of 52 children with newly identified islet cell aAb positivity already had first-phase insulin responses (FPIRs) below the fifth percentile (5). These data suggest that loss of early C-peptide responses are among the first and most identifiable defects in at-risk individuals. As to how these defects occur are a matter of speculation. It is possible that immune activation caused a quantum 
A

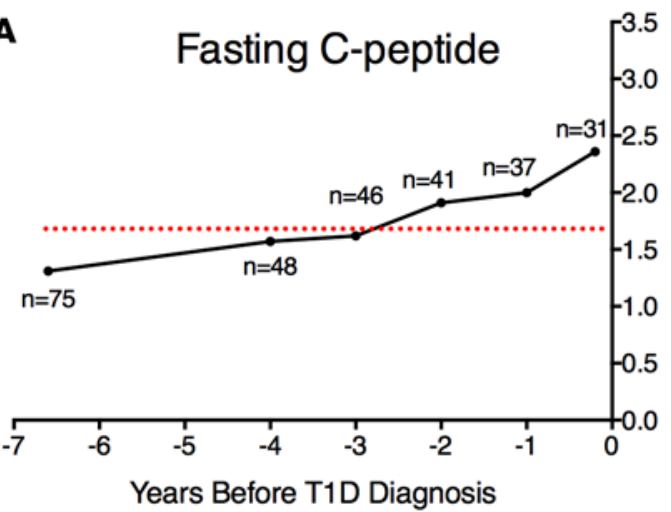

C

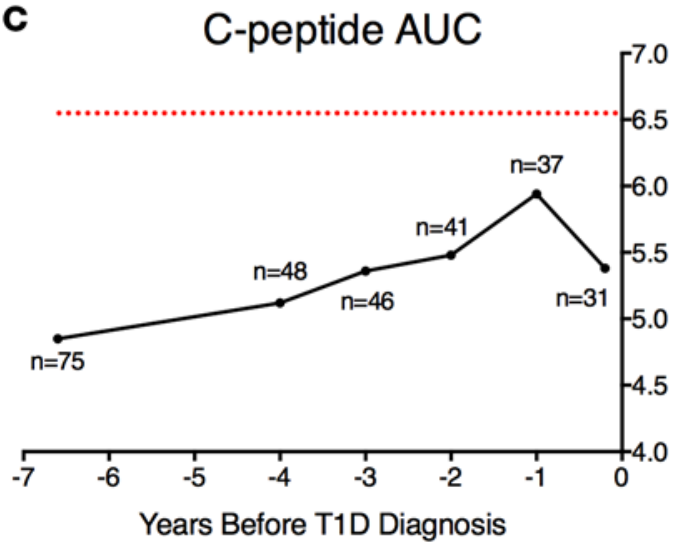

$\mathbf{E}$
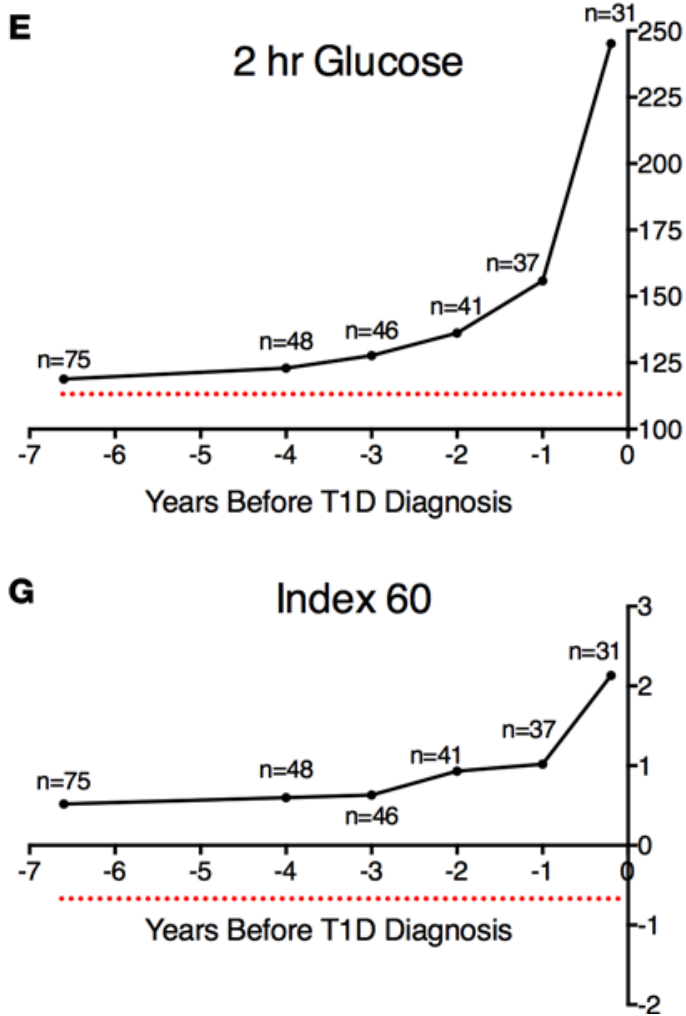

D

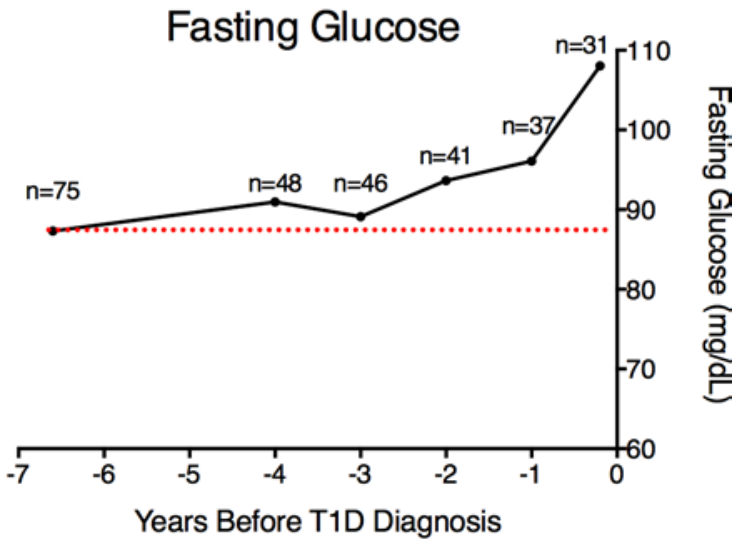

$\mathbf{F}$

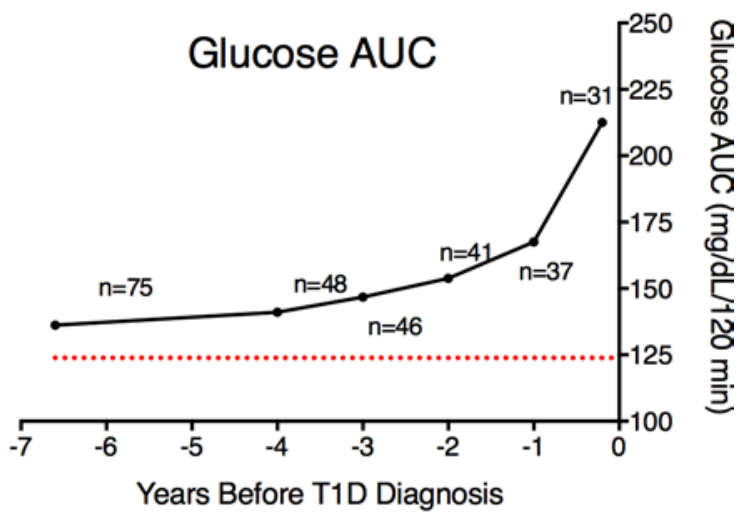

Figure 2. Longitudinal patterns of metabolic decline in Progressors $\geq \mathbf{5}$. Shown are the mean values for the fasting C-peptide (A), early C-peptide response (B), C-peptide AUC (C), fasting glucose (D), 2-hour glucose (E), glucose AUC, (F) and Index60 (C) at baseline and then each year ( \pm 6 months) from 4 years to diagnosis for Progressors $\geq 5$. Also shown for reference are mean values of the $a A b$ - individuals at baseline (red dotted line). Note that the $a A b-c o n t r o l$ group $(n=144)$ was studied at a single time point, and the dotted line indicates the value obtained at this single time point. The number of Progressors $\geq 5$ with available data at each time point is indicated. 
Table 2. Longitudinal trends of metabolic progression to T1D in Progressors $\geq 5$

\begin{tabular}{|c|c|c|c|c|c|c|c|c|c|}
\hline & \multicolumn{3}{|c|}{ Baseline to 4 Years Before Diagnosis $(n=48)$} & \multicolumn{3}{|c|}{4 Years to 1 Year Before Diagnosis $(n=30)$} & \multicolumn{3}{|c|}{1 Year Before to Diagnosis $(n=27)$} \\
\hline & $\begin{array}{l}\text { Abs Change } \\
\text { Median (IQQR) }\end{array}$ & $\begin{array}{c}\text { \%Change } \\
\text { Median (IQR) }\end{array}$ & P value ${ }^{A}$ & $\begin{array}{l}\text { Abs Change } \\
\text { Median (IQR) }\end{array}$ & $\begin{array}{c}\text { \%Change } \\
\text { Median (IQR) }\end{array}$ & $P$ value $^{A}$ & $\begin{array}{l}\text { Abs Change } \\
\text { Median (IQQR) }\end{array}$ & $\begin{array}{c}\text { \%Change } \\
\text { Median (IQQR) }\end{array}$ & $P$ value ${ }^{A}$ \\
\hline $\begin{array}{l}\text { Fasting } \\
\text { C-peptide } \\
\text { (ng/ml) }\end{array}$ & $\begin{array}{c}0.23 \\
(-0.11 \text { to } 0.52)\end{array}$ & $\begin{array}{c}21.61 \\
(-7.38 \text { to } 51.31)\end{array}$ & 0.001 & $\begin{array}{c}0.14 \\
(-0.06 \text { to } 0.58)\end{array}$ & $\begin{array}{c}11.00 \\
\text { (-2.00 to } 37.00)\end{array}$ & 0.01 & $\begin{array}{c}0.13 \\
(-0.23 \text { to } 0.81)\end{array}$ & $\begin{array}{c}5.00 \\
(-9.00 \text { to } 30.00)\end{array}$ & 0.13 \\
\hline $\begin{array}{l}\text { Early } \\
\text { C-peptide } \\
\text { Response } \\
\text { (ng/ml) }\end{array}$ & $\begin{array}{c}-0.18 \\
(-0.92 \text { to } 0.88)\end{array}$ & $\begin{array}{c}-5.33 \\
(-30.18 \text { to } 33.58)\end{array}$ & 0.76 & $\begin{array}{c}-0.43 \\
(-1.23 \text { to } 0.40)\end{array}$ & $\begin{array}{c}-28.00 \\
(-42.00 \text { to } 11.00)\end{array}$ & 0.11 & $\begin{array}{c}-0.44 \\
(-2.05 \text { to }-0.02)\end{array}$ & $\begin{array}{c}-35.00 \\
(-47.00 \text { to }-2.00)\end{array}$ & $<0.001$ \\
\hline $\begin{array}{l}\text { C-Peptide AUC } \\
(\mathrm{mg} / \mathrm{dl} / 120 \\
\mathrm{min})\end{array}$ & $\begin{array}{c}0.11 \\
(-0.55 \text { to } 1.20)\end{array}$ & $\begin{array}{c}2.34 \\
(-10.97 \text { to } 32.96)\end{array}$ & 0.32 & $\begin{array}{c}-0.05 \\
(-0.57 \text { to } 0.69)\end{array}$ & $\begin{array}{c}-1.00 \\
(-14.00 \text { to } 15.00)\end{array}$ & 0.90 & $\begin{array}{c}-0.58 \\
(-1.58 \text { to } 0.09)\end{array}$ & $\begin{array}{c}-12.00 \\
(-22.00 \text { to } 2.00)\end{array}$ & 0.01 \\
\hline $\begin{array}{l}\text { Fasting } \\
\text { Glucose } \\
\text { (mg/dl) }\end{array}$ & $\begin{array}{c}2.50 \\
(-4.00 \text { to } 8.00)\end{array}$ & $\begin{array}{c}2.89 \\
(-4.19 \text { to } 9.82)\end{array}$ & 0.02 & $\begin{array}{c}5.00 \\
(1.00 \text { to } 12.00)\end{array}$ & $\begin{array}{c}5.00 \\
(1.00 \text { to } 12.00)\end{array}$ & 0.01 & $\begin{array}{c}6.00 \\
(0.00 \text { to } 26.00)\end{array}$ & $\begin{array}{c}7.00 \\
\text { (0.00 to } 25.00)\end{array}$ & $<0.001$ \\
\hline Glucose AUC & $\begin{array}{c}7.17 \\
(-11.50 \text { to } 28.58)\end{array}$ & $\begin{array}{c}5.86 \\
(-7.35 \text { to } 22.59)\end{array}$ & 0.03 & $\begin{array}{c}14.21 \\
(-11.92 \text { to } 44.75)\end{array}$ & $\begin{array}{c}11.00 \\
(-8.00 \text { to } 30.00)\end{array}$ & 0.01 & $\begin{array}{c}39.67 \\
\text { (20.17 to } 64.92)\end{array}$ & $\begin{array}{c}27.00 \\
(12.00 \text { to } 44.00)\end{array}$ & $<0.001$ \\
\hline Index60 & $\begin{array}{c}0.15 \\
(-0.24 \text { to } 0.48)\end{array}$ & $\begin{array}{c}-11.28 \\
(-91.59 \text { to } 40.66)\end{array}$ & 0.11 & $\begin{array}{c}0.34 \\
(-0.09 \text { to } 0.97)\end{array}$ & $\begin{array}{c}1.00 \\
(-55.00 \text { to } \\
54.00)\end{array}$ & 0.004 & $\begin{array}{c}1.09 \\
\text { (0.68 to } 1.68)\end{array}$ & $\begin{array}{c}70.00 \\
(-9.00 \text { to } 224.00)\end{array}$ & $<0.001$ \\
\hline
\end{tabular}

AWilcoxon's signed-rank tests were used to compare absolute changes between the time intervals. Abs, absolute; IQR, interquartile range; AUC, area under the curve; T1D, type 1 diabetes.

loss of $\beta$ cell function just prior to the baseline observation. On the other hand, loss of $\beta$ cell function could conceivably have been gradual, but it would have taken time to evolve to such a marked abnormality. Yet another explanation is that there is a genetic proclivity for $\beta$ cell dysfunction that could be independent of the immunopathogenesis of T1D. This would suggest a 2-component causal model for T1D comprising constitutional metabolic impairments that act in concert with autoimmunity.

Second metabolic phase. The second metabolic phase was characterized by a pattern of more gradual deterioration. An important consideration is whether this phase represented a period of immune regulation or reflects a relapsing and remitting course of autoimmunity in T1D (6). Alternatively, these data could represent an adaptive period of pancreatic $\beta$ cell function, characterized by notable changes in the architecture of C-peptide responses with relative preservation of glycemia. Prior longitudinal analysis of DPT-1 data revealed an increase in the late C-peptide OGTT response during T1D progression (2). Similarly, we observed an unexpected increase in the C-peptide AUC in the setting of continued and significant defects in the early C-peptide response. This finding is not dissimilar to what is observed in the prediabetic phase of type 2 diabetes (T2D) (7). Whether this response represents the secretory profile of sick, damaged, or dedifferentiated $\beta$ cells or an adaptive response from subpopulations of remaining $\beta$ cells that have escaped immune destruction $(8,9)$ is uncertain but warrants further investigation.

Third metabolic phase. Beginning around 2 years prior to the T1D diagnosis, there was evidence for a third metabolic phase, characterized by significant reductions in C-peptide secretion and rising glycemia. The majority of published studies have focused analyses around this particular time point. For example, previous longitudinal analyses of $\mathrm{aAb}+$ individuals followed in the DPT-1 study revealed decreased early C-peptide responses during OGTTs and a gradual pattern of worsening glycemia around 2 years prior to T1D diagnosis. Analysis of the DPT-1 cohort also identified reduced $\beta$ cell glucose sensitivity around 1.5 years prior to T1D onset $(2,3)$. Our study adds to the previous literature by significantly extending the length of follow-up in the prediagnostic period and by analysis of participants more heterogeneous in age and geography.

An outstanding question is the pathologic basis for the marked acceleration in $\beta$ cell dysfunction during this terminal phase. The acceleration of metabolic decline in T1D could be similar to the accelerated pathologic decline at the end stage of other diseases, representing an irremediable threshold effect from chronic 
Table 3. Comparison of longitudinal trends between Progressors $\geq 5$ and Progressors $<5$

\begin{tabular}{|c|c|c|c|c|c|c|}
\hline \multirow[b]{2}{*}{ Variable } & \multicolumn{2}{|c|}{3 Years Prior to T1D ( \pm 6 months) } & \multicolumn{2}{|c|}{2 Years Prior to T1D ( \pm 6 months) } & \multicolumn{2}{|c|}{1 year Prior to T1D ( \pm 6 months) } \\
\hline & $\begin{array}{c}\text { Progressors } \geq 5 \\
(n=29) \text { Mean (SD) }\end{array}$ & $\begin{array}{c}\text { Progressors }<5 \\
(n=72) \text { Mean (SD) }\end{array}$ & $\begin{array}{c}\text { Progressors } \geq 5 \\
(n=29) \text { Mean (SD) }\end{array}$ & $\begin{array}{c}\text { Progressors }<5 \\
(n=72) \text { Mean (SD) }\end{array}$ & $\begin{array}{c}\text { Progressors } \geq 5 \\
(n=29) \text { Mean (SD) }\end{array}$ & $\begin{array}{c}\text { Progressors }<5 \\
(n=72) \text { Mean (SD) }\end{array}$ \\
\hline $\begin{array}{l}\text { Fasting C-peptide } \\
(\mathrm{ng} / \mathrm{ml})\end{array}$ & $1.67(0.92)$ & $1.43(0.64)$ & $1.88(0.88)$ & $1.59(0.81)$ & $1.90(0.99)$ & $1.68(0.75)$ \\
\hline $\begin{array}{l}\text { Early C-peptide } \\
\text { Response (ng/ml) }\end{array}$ & $2.89(1.49)$ & $3.07(1.66)$ & $2.74(1.55)$ & $2.64(1.50)$ & $2.75(1.80)$ & $2.61(1.37)$ \\
\hline $\begin{array}{l}\text { Fasting Glucose } \\
\text { (mg/dl) }\end{array}$ & $89.14(10.96)$ & $89.43(9.11)$ & $93.55(11.22)$ & $90.01(11.89)$ & $94.69(12.47)$ & $92.83(11.65)$ \\
\hline $\begin{array}{l}\text { 2-hour Glucose } \\
\text { (mg/dl) }\end{array}$ & $127.66(25.3)$ & $129.64(28.12)$ & $142.45(34.24)$ & $136.35(31.87)$ & $153.14(33.31)$ & $151.13(38.78)$ \\
\hline Glucose AUC & $147.27(20.59)$ & $143.37(21.59)$ & $158.15(27.27)$ & $152.36(24.92)$ & $166.14(25.54)$ & $161.7(26.24)$ \\
\hline Index60A & $0.69(0.91)$ & $0.69(0.79)$ & $0.90(0.94)$ & $0.92(0.85)$ & $1.03(1.06)$ & $1.14(0.93)$ \\
\hline
\end{tabular}

No significant differences between the groups were found at any time point using Student's $t$ tests or multivariate linear regression with adjustment for age and BMI $Z$ scores. Analysis limited to Progressors $\geq 5$ and Progressors $<5$ who had all 3 OGTTs. Andex60 values were log transformed for comparisons. AUC, area under the curve; SD, standard deviation; T1D, type 1 diabetes.

$\beta$ cell loss. Another possibility is these patterns result from a second immunologic hit that precedes this terminal stage of $\beta$ cell decline. Notwithstanding this uncertainty, defining the actual reduction in $\beta$ cell mass needed for a diagnosis of T1D has been challenging and likely differs significantly by individual. Classic natural history models invoke a near-complete loss of $\beta$ cell mass by the time of diagnosis. However, more recent analysis of pancreatic sections from persons with established T1D have documented the presence of $\beta$ cells many years after the diagnosis of T1D, challenging this classic notion. Moreover, $\beta$ cell mass has been shown to be quite variable in those with established disease, while data from $a A b+$ donors from the $\mathrm{nPOD}$ collection suggest that $\beta$ cell mass is relatively preserved in early-stage disease (10-12). Thus, whether this terminal decline represents primarily a loss of $\beta$ cell function or mass remains unclear. Interestingly, results from the Diabetes Virus Detection (DiViD) study demonstrated that ex vivo recovery of islets obtained via pancreatic biopsies from individuals with recent-onset T1D led to improved insulin secretory profiles. While insulin secretion remained lower than islets from nondiabetic donors, these data nonetheless indicate that a component of $\beta$ cell function (as opposed to mass) is reparable in the short term (13).

Our study is the first to our knowledge to identify the presence of $\beta$ cell dysfunction so far in advance of the diagnosis of diabetes. The observation that $\beta$ cell function was already reduced so many years in advance of the diagnosis raises the intriguing possibility that genetic defects in either $\beta$ cell function, mass, or survival, are present in some individuals who develop autoimmunity and T1D. Gene variants associated with the development of T1D have been classically associated with altered immune function and self-tolerance. However, increased expression of a number of these classical T1D risk genes has been identified in cytokinestressed $\beta$ cells, while upregulation of many of these genes has been linked with $\beta$ cell inflammatory and apoptotic signaling $(14,15)$. In contrast to T1D, GWAS studies in T2D have largely identified variants associated with impairments in $\beta$ cell function (16). However, few studies have analyzed whether genes associated with impaired $\beta$ cell function in T2D might be similarly associated with T1D. One notable exception is transcription factor 7-like 2 (TCF7L2). Polymorphisms in TCF7L2 have been identified in T2D cohorts and are associated with impaired insulin secretion and reduced glucagon suppression $(16,17)$. Recently, variants of TCF7L2 were found to be more frequent in a subset of individuals diagnosed with T1D in the setting of fewer aAbs and decreased frequency of lower-risk HLA haplotypes (18-20), suggesting that individuals with TCF7L2 risk alleles may cross the threshold of diagnosis in the presence of a lower immune burden.

In further support of the notion that $\beta$ cell susceptibility may contribute to the development of T1D, a number of studies have analyzed insulin secretion in $\mathrm{aAb}$ - relatives of T1D index cases and found varying degrees of $\beta$ cell dysfunction (21-23). While these data suggest the possibility that T1D kindreds may have preexisting impairments in $\beta$ cell function, they also highlight one potential limitation of our control group. We utilized data from first-, second-, and third-degree relatives who underwent oral glucose tolerance testing 
around the time of a negative aAb screen. While we still observed large differences between Progressors $\geq 5$ and the control group, it is possible that even more substantial differences would be present using a control group comprising individuals unrelated to persons with T1D.

Finally, it is necessary to consider how representative Progressors $\geq 5$ are of all individuals diagnosed with T1D. Progressors $\geq 5$ comprised $13.7 \%$ of analyzed diabetes cases within the cohort. Because $a A b+$ individuals are enrolled into TrialNet in a cross-sectional manner, we do not know the true duration of $\mathrm{aAb}$ positivity prior to the initial $\mathrm{aAb}+$ screen. As such, this likely represents a minimum estimate of the number of individuals who have aAb positivity for 5 or more years before T1D diagnosis. Metabolic dysfunction may have been present for a substantially longer period of time in some Progressors $\geq 5$ and certainly may have been present for longer in Progressors $<5$. However, a striking finding of our analysis was the great deal of similarity in the degree and patterns of $\beta$ cell dysfunction during the last 3 years before diagnosis between Progressors $\geq 5$ and Progressors $<5$. These findings suggest that, despite differences in age and potentially length of $\mathrm{aAb}$ positivity, there may be a characteristic pattern of decline in $\beta$ cell function within 3 years of clinical diagnosis. The number of subjects who had metabolic data at each of the specified time points shown in Table 3 and Supplemental Table 3 was limited. Therefore, it would be of interest to determine whether this similar pattern can be corroborated in other populations, including birth cohorts that are followed both before and after seroconversion.

Taken together, these data provide insight into the chronicity of $\beta$ cell dysfunction in T1D and provide what we believe to be a new nomenclature for describing 3 distinct metabolic phases of T1D evolution. We also establish a hierarchy of $\beta$ cell decline that should be considered for the purposes of risk stratification and when designing interventions that test disease-modifying therapies.

\section{Methods}

$a A b+$ participants and follow-up. Criteria for entry into the TrialNet PTP cohort have been previously described in detail (24). In brief, nondiabetic first-, second-, or third-degree relatives of individuals with T1D (proband) were screened for the presence of aAbs specific for pancreatic islets. aAb status was assessed using procedures outlined previously in the Diabetes Antibody Standardization Program (25). Initial testing for glutamic acid decarboxylase 65 (GAD65), microinsulin aAb assay (mIAA), or islet-antigen 2 (IA-2/ICA512) aAbs was followed by measurement of islet cell (ICA) or zinc transporter 8 (ZnT8) aAbs if any one initial test was positive (26). ZnT8 was measured in a limited group of PTP participants beginning in 2004 (27), and measurement of ZnT8 for the entire PTP cohort began in 2012. From 2004 through $12 / 31 / 2016$, a total of 180,452 individuals were screened for the presence of these aAbs against $\beta$ cell antigens. The majority $(n=174,929)$ were identified as aAb-, whereas 5,523 individuals $(3 \%)$ were identified as either confirmed single or multiple $\mathrm{aAb}+$ and enrolled for longitudinal monitoring (Figure 1). Longitudinal monitoring of $\mathrm{aAb}+$ individuals consisted of semiannual or annual study visits that included a standard protocol 2-hour OGTT (28). Glucose was measured using the glucose oxidase method (29); C-peptide was measured using a 2-site immunoenzymometric assay performed on a Tosoh II 600 autoanalyzer (30). Diabetes was diagnosed according to American Diabetes Association criteria (31). A total of 1,032 subjects within the PTP cohort have been diagnosed with T1D; 483 were excluded from this analysis. These included subjects with a diabetic OGTT at study entry, incomplete baseline OGTT data, previous participation in the DPT-1 study, or current participation in a prevention trial.

$a A b$-participants. Since 2008, 144 relatives of T1D patients having no positive aAbs in the first screening sample were selected for enrollment into an $\mathrm{aAb}$ - control group. These individuals underwent a 2-hour OGTT shortly after the negative aAb screening.

Statistics. To compare demographic characteristics between groups, Pearson's $\chi^{2}$ test was used for categorical data. For comparisons of continuous data, ANOVA tests were used to compare means, while Kruskal-Wallis tests were used to compare medians. Metabolic parameters were derived from OGTTs. The early C-peptide response was defined as the difference between the 30- and 0-minute C-peptide values. Glucose and C-peptide AUCs were defined as total area under the 2-hour OGTT curve, calculated using the trapezoid rule, and then divided by 120 minutes. The Index60 was calculated according to the following formula: Index60 $=(0.3695 \times \log$ fasting C-peptide $)+(0.0165 \times$ 60 -minute glucose $)-(0.3644 \times 60$-minute C-peptide $)(32)$. Metabolic parameters between groups were compared using Student's $t$ tests and a multivariate linear regression model with adjustment for age and BMI $Z$ score. Index60 values were log transformed before comparisons. Changes in metabolic 
parameters over time were assessed using a general linear mixed model. The absolute change between 2 time points was calculated as the value at the later time point minus the value at the earlier time point; the percentage change between 2 time points was calculated as the difference between 2 time points divided by the value at the earlier time point, which was multiplied by 100 . Paired $t$ tests were used to assess significant change in metabolic parameters between 2 time points. When the sample size was less than 30, a nonparametric Wilcoxon's signed-rank test was used. Unpaired $t$ tests and MannWhitney tests were used to analyze between-group differences (Progressors $\geq 5$ vs. Progressors $<5$ ). Statistical analyses were performed with SAS (version 9.4; SAS Institute). All tests of significance were 2 tailed, and $P$ values $<0.05$ were considered statistically significant. $P$ values were not adjusted for multiple testing, as our a priori intent was to test each variable independently.

Study approval. All human studies were approved by participating institutional review boards and written informed consent was received from participants prior to inclusion in the study.

\section{Author contributions}

CEM, JMS, and PX directed the conception and design of the study, data analysis and interpretation, collection and assembly of data, and manuscript writing. PX and SG provided statistical analysis and contributed to the collection and assembly of data, and manuscript writing. EKS, LAD, HMI, AKS, JPP, and JPK contributed to the interpretation of data and manuscript writing.

\section{Acknowledgments}

The Type 1 Diabetes TrialNet Study Group is a clinical trials network currently funded by the NIH through the National Institute of Diabetes and Digestive and Kidney Diseases, the National Institute of Allergy and Infectious Diseases, and The Eunice Kennedy Shriver National Institute of Child Health and Human Development, through the cooperative agreements U01 DK061010, U01 DK061034, U01 DK061042, U01 DK061058, U01 DK085461, U01 DK085465, U01 DK085466, U01 DK085476, U01 DK085499, U01 DK085509, U01 DK103180, U01 DK103153, U01 DK103266, U01 DK103282, U01 DK106984, U01 DK106994, U01 DK107013, U01 DK107014, UC4 DK106993, and the Juvenile Diabetes Research Foundation International (JDRF). See supplemental acknowledgments for consortium details. The contents of this Article are solely the responsibility of the authors and do not necessarily represent the official views of the NIH or the JDRF. This work was also partially supported by NIH grants R01 DK093954 and UC4 DK 104166 (to C. Evans-Molina), K08DK103983 (to E.K. Sims), VA Merit Award I01BX001733 (to C. Evans-Molina), a JDRF Strategic Research Agreement (to C. Evans-Molina), and gifts from the Sigma Beta Sorority, the Ball Brothers Foundation, the George and Frances Ball Foundation, and the Holiday Management Foundation. See supplemental acknowledgments for The Type 1 Diabetes TrialNet Study Group details.

Address correspondence to: Carmella Evans-Molina, Indiana University School of Medicine, 635 Barnhill Drive MS 2031A, Indianapolis, Indiana, USA. Phone: 317.274.4145; Email: cevansmo@iu.edu.

1. Sosenko JM, et al. Acceleration of the loss of the first-phase insulin response during the progression to type 1 diabetes in diabetes prevention trial-type 1 participants. Diabetes. 2013;62(12):4179-4183.

2. Sosenko JM, et al. Trends of earlier and later responses of C-peptide to oral glucose challenges with progression to type 1 diabetes in diabetes prevention trial-type 1 participants. Diabetes Care. 2010;33(3):620-625.

3. Ferrannini E, Mari A, Nofrate V, Sosenko JM, Skyler JS, DPT-1 Study Group. Progression to diabetes in relatives of type 1 diabetic patients: mechanisms and mode of onset. Diabetes. 2010;59(3):679-685.

4. Battaglia M, et al. Understanding and preventing type 1 diabetes through the unique working model of TrialNet. Diabetologia. 2017;60(11):2139-2147.

5. Keskinen P, et al. First-phase insulin response in young healthy children at genetic and immunological risk for type I diabetes. Diabetologia. 2002;45(12):1639-1648.

6. van Megen KM, et al. Relapsing/remitting type 1 diabetes. Diabetologia. 2017;60(11):2252-2255.

7. Fonseca VA. Defining and characterizing the progression of type 2 diabetes. Diabetes Care. 2009;32 Supp1 2:S151-S156.

8. Dorrell C, et al. Human islets contain four distinct subtypes of $\beta$ cells. Nat Commun. 2016;7:11756.

9. Rui J, Deng S, Arazi A, Perdigoto AL, Liu Z, Herold KC. $\beta$ Cells that resist immunological attack develop during progression of autoimmune diabetes in NOD mice. Cell Metab. 2017;25(3):727-738.

10. Campbell-Thompson M, et al. Insulitis and $\beta$-cell mass in the natural history of type 1 diabetes. Diabetes. 2016;65(3):719-731.

11. Wagner R, et al. Lack of immunohistological changes in the islets of nondiabetic, autoimmune, polyendocrine patients with beta-selective GAD-specific islet cell antibodies. Diabetes. 1994;43(7):851-856. 
12. Rodriguez-Calvo T, et al. Increase in pancreatic proinsulin and preservation of $\beta$-cell mass in autoantibody-positive donors prior to type 1 diabetes onset. Diabetes. 2017;66(5):1334-1345.

13. Krogvold L, et al. Function of isolated pancreatic islets from patients at onset of type 1 diabetes: Insulin secretion can be restored after some days in a nondiabetogenic environment in vitro: results from the DiViD study. Diabetes. 2015;64(7):2506-2512.

14. Marroqui L, et al. TYK2, a candidate gene for type 1 diabetes, modulates apoptosis and the innate immune response in human pancreatic $\beta$-cells. Diabetes. 2015;64(11):3808-3817.

15. Eizirik DL, et al. The human pancreatic islet transcriptome: expression of candidate genes for type 1 diabetes and the impact of pro-inflammatory cytokines. PLoS Genet. 2012;8(3):e1002552.

16. Florez JC. Newly identified loci highlight beta cell dysfunction as a key cause of type 2 diabetes: where are the insulin resistance genes? Diabetologia. 2008;51(7):1100-1110.

17. Shah M, et al. TCF7L2 Genotype and $\alpha$-cell function in humans without diabetes. Diabetes. 2016;65(2):371-380

18. Redondo MJ, et al. Association of TCF7L2 variation with single islet autoantibody expression in children with type 1 diabetes BMJ Open Diabetes Res Care. 2014;2(1):e00008.

19. Redondo MJ, Grant SF, Davis A, Greenbaum C, T1D Exchange Biobank. Dissecting heterogeneity in paediatric type 1 diabetes: association of TCF7L2 rs7903146 TT and low-risk human leukocyte antigen (HLA) genotypes. Diabet Med. 2017;34(2):286-290.

20. Redondo MJ, et al. TCF7L2 genetic variants contribute to phenotypic heterogeneity of type 1 diabetes. Diabetes Care. 2018;41(2):311-317.

21. Vialettes B, Mattei-Zevaco C, Badier C, Ramahandridona G, Lassmann-Vague V, Vague P. Low acute insulin response to intravenous glucose. A sensitive but non-specific marker of early stages of type 1 (insulin-dependent) diabetes. Diabetologia. 1988;31(8):592-596

22. Carel JC, Boitard C, Bougnères PF. Decreased insulin response to glucose in islet cell antibody-negative siblings of type 1 diabetic children. J Clin Invest. 1993;92(1):509-513.

23. Siewko K, et al. Prognostic markers for the development of type 1 diabetes in first-degree relatives of diabetic patients. Endokrynol Pol. 2014;65(3):176-180.

24. Skyler JS, et al. Type 1 Diabetes TrialNet--an international collaborative clinical trials network. Ann N Y Acad Sci. 2008;1150:14-24.

25. Vehik K, et al. Development of autoantibodies in the TrialNet Natural History Study. Diabetes Care. 2011;34(9):1897-1901.

26. Mahon JL, et al. The TrialNet Natural History Study of the Development of Type 1 Diabetes: objectives, design, and initial results. Pediatr Diabetes. 2009;10(2):97-104.

27. Yu L, et al. Zinc transporter-8 autoantibodies improve prediction of type 1 diabetes in relatives positive for the standard biochemical autoantibodies. Diabetes Care. 2012;35(6):1213-1218.

28. Greenbaum CJ, et al. Mixed-meal tolerance test versus glucagon stimulation test for the assessment of beta-cell function in therapeutic trials in type 1 diabetes. Diabetes Care. 2008;31(10):1966-1971.

29. Sosenko JM, et al. A comparison of the baseline metabolic profiles between Diabetes Prevention Trial-Type 1 and TrialNet Natural History Study participants. Pediatr Diabetes. 2011;12(2):85-90.

30. Hao W, Gitelman S, DiMeglio LA, Boulware D, Greenbaum CJ, Type 1 Diabetes TrialNet Study Group. Fall in C-peptide during first 4 years from diagnosis of type 1 diabetes: Variable relation to age, HbA1c, and insulin dose. Diabetes Care. 2016;39(10):1664-1670.

31. American Diabetes Association. Standards of medical care in diabetes--2014. Diabetes Care. 2014;37 Suppl 1:S14-S80.

32. Sosenko JM, et al. A new approach for diagnosing type 1 diabetes in autoantibody-positive individuals based on prediction and natural history. Diabetes Care. 2015;38(2):271-276. 